\title{
Avaliação da capacidade de gestão descentralizada da vigilância epidemiológica no estado da Bahia
}

\author{
Evaluation of the capability of decentralized management \\ of epidemiological surveillance in the state of Bahia
}

\author{
Silvone Santa Bárbara da Silva Santos ${ }^{1}$ \\ Cristina Maria Meira de Melo ${ }^{2}$ \\ Heloniza Oliveira Gonçalves Costa ${ }^{2}$ \\ Oswaldo Yoshimi Tanaka ${ }^{3}$ \\ Fernanda Mota Ramos ${ }^{2}$ \\ Mário César Carneiro de Santana ${ }^{2}$ \\ Bianca Gonzaga Trindade ${ }^{2}$
}

${ }^{1}$ Departamento de Saúde, Universidade Estadual de Feira de Santana. Avenida Transnordestina s/n, Novo Horizonte. 44036-900 Feira de Santana Bahia. silvone.s@uefs.br

${ }^{2}$ Departamento de Enfermagem Comunitária, Escola de Enfermagem, Universidade Federal da Bahia.

${ }^{3}$ Faculdade de Saúde Pública, Universidade de São Paulo.

\begin{abstract}
Evaluative research into the capability of decentralized management of epidemiological vigilance $(E V)$ was conducted in the operational, organizational and sustainable dimensions in the state of Bahia, Brazil. The quantitative approach was used in the construction of a baseline, with primary data obtained through an online questionnaire answered by thirty-eight municipal EV managers. In the qualitative approach to analyze the context and assess the management capability of municipalities in two case studies, techniques adapted to the analysis of discursive practices were used. This was done through semi-structured interviews with managers of regional and municipal government, health workers and representatives of the municipal health council. The case studies showed that the municipality with enhanced management capability is that in which the manager has the greatest potential of using the resources of his position, in addition to his ability to control, negotiate and coordinate with other actors. Due to decentralization of EV, considering the shared nature of management between the three spheres of government, there is a marked variation in the management capability of municipalities, determined by social, econom$i c$, political inequalities and management mechanisms adopted.
\end{abstract}

Key words Management capacity, Evaluation, Decentralization, Epidemiological surveillance
Resumo Pesquisa avaliativa da capacidade de gestão descentralizada da vigilância epidemiológica (VE) no estado da Bahia, Brasil, em três dimensões: operacional, organizacional e da sustentabilidade. A abordagem quantitativa é usada na construção de uma linha de base, com dados primários obtidos através de questionário on line respondido por 38 gestores da VE municipal. $\mathrm{Na}$ abordagem qualitativa para análise de contexto $e$ para avaliar a capacidade de gestão em dois municípios casos utiliza-se das técnicas adaptadas da análise das práticas discursivas, através de entrevistas semiestruturadas com os gestores da secretaria estadual e municipais da Saúde, trabalhadores e representantes do conselho municipal de saúde. Dados secundários foram obtidos através da análise documental. Os estudos de caso demonstram que o município com melhor capacidade de gestão é aquele cujo gestor tem maior potencial de operar os recursos oriundos da posição que ocupa, somada à sua capacidade de controlar, negociar e articular. Em decorrência da descentralização da VE, considerando a natureza compartilhada da gestão entre as três esferas de governo, existe uma expressiva variação na capacidade de gestão dos municípios, determinada por desigualdades sociais, econômicas, políticas e dos mecanismos de gestão adotados.

Palavras-chave Capacidade de gestão, Avaliação, Descentralização, Vigilância epidemiológica 


\section{Introdução}

Para responder às perguntas sobre qual é a capacidade de gestão da Vigilância Epidemiológica (VE) nos municípios baianos e por que estes municípios possuem graus diferenciados de capacidade de gestão é que se desenvolveu a presente pesquisa. O referencial teórico está embasado na evolução histórica das concepções e das práticas da vigilância epidemiológica, descentralização, gestão e avaliação em saúde.

Neste estudo elaborou-se uma concepção de capacidade de gestão compreendida como o potencial que tem um ator em operar os recursos existentes e em adquirir novos recursos (normativos, técnicos, materiais, políticos, relacionais e de informação), bem como a sua capacidade para mobilizar os recursos oriundos da posição que ocupa de modo a controlar, negociar e articular com outros atores no processo de tomada de decisão. Assim, a capacidade de gestão refere-se ao exercício do poder e à sua legitimidade ${ }^{1-4}$.

\section{Metodologia}

Esta é uma pesquisa avaliativa quantitativa/qualitativa, para a construção de uma linha de base e para estudos de casos múltiplos dos municípios melhor avaliados.

Adotam-se como definições-guia nesta avaliação:

. A Vigilância Epidemiológica: "o conjunto de ações que proporcionam o conhecimento, a detecção e a prevenção de qualquer mudança nos fatores determinantes e condicionantes da saúde individual ou coletiva, e tem como finalidade recomendar e adotar as medidas de prevenção e controle de doenças ou agravos"s.

. Capacidade de gestão descentralizada da VE: a capacidade que tem um ou diversos atores para mobilizar os recursos disponíveis e ou buscar novos, sejam estes normativos, materiais, relacionais, de informação ou técnicos, bem como recursos oriundos da posição que ocupam (poder) para negociar com outros atores, com vistas ao desenvolvimento de ações que impliquem na melhoria dos indicadores de saúde da população e na sustentação dos resultados obtidos.

Considerando os objetivos, a extensão e o tipo da pesquisa, a metodologia está organizada em dois momentos: o primeiro, com enfoque quantitativo, e o segundo, com enfoque qualitativo. Apesar de estarem sendo apresentados didaticamente os dois momentos, cabe ressaltar que foi feita uma avaliação por triangulação de métodos, ao se entrelaçar várias estratégias de pesquisa visando apreender as dimensões qualitativas e quantitativas do objeto de estudo ${ }^{6}$.

Para a construção da linha de base avaliativa, o campo de investigação é constituído pelos municípios do estado da Bahia, numa amostra por cluster. O cluster primário são as macrorregiões e o cluster secundário os municípios, a partir do Plano Diretor de Regionalização. Foram selecionados 108 municípios, sendo 38 os amostrados, distribuídos entre as nove macrorregiões do estado. Muito embora a seleção inicial tenha sido de doze municípios por macrorregião, o maior número de municípios amostrados concentrou-se na macrorregião Nordeste. Nas macrorregiões Centro-Leste e Leste, que possuem os municípios mais populosos, obteve-se um menor retorno do questionário. No entanto, a capital do estado, inserida na macrorregião Leste, bem como o segundo maior município do estado, que integra a macrorregião Centro-Leste, estão entre os municípios amostrados. Os municípios foram selecionados para a construção da linha de base avaliativa em função dos seguintes critérios iniciais: município referência macrorregional; município referência ou não de macrorregião e referência microrregional; município referência ou não de macrorregião, referência ou não de microrregional e sede da Diretoria Regional de Saúde (Dires); município referência ou não de macrorregião, referência ou não de microrregional e sede ou não de Dires e com a gestão plena do sistema de saúde.

Os atores são estratificados em grupos de representação e selecionados intencionalmente, de acordo com os objetivos da pesquisa. Para a construção da linha de base avaliativa os gestores da VE municipal responderam a um questionário via on line, formulado contemplando as dimensões do triângulo de governo ${ }^{2}$, conforme Guimarães et al. ${ }^{3}$ e Barreto e Guimarães ${ }^{4}$, e disponibilizado por sítio institucional da Secretaria da Saúde do Estado da Bahia (Sesab). O questionário toma como base os indicadores construídos através de oficinas de trabalho, junto aos gestores e técnicos da Divep e ao grupo de pesquisa Gerir/EEUFBA. As oficinas possibilitaram a revisão de indicadores propostos inicialmente, além de permitir a inserção de gestores estaduais no processo. O projeto foi aprovado pelo Comitê de Ética em Pesquisa da Sesab.

A análise do contexto foi construída a partir das enunciações extraídas das entrevistas semiestruturadas de seis gestores da VE estadual. 
Para os casos foram selecionados dois municípios: um, cuja linha de base avaliativa apontou a melhor capacidade de gestão da VE; outro, cuja linha de base avaliativa apontou a menor capacidade de gestão da VE. Foram entrevistados o gestor máximo da secretaria municipal da saúde (SMS), o coordenador da VE e o da Atenção Básica; trabalhadores da vigilância epidemiológica do âmbito central da SMS; trabalhadores da unidade básica de saúde e representantes dos usuários no Conselho Municipal de Saúde (CMS), num total de onze entrevistados.

Dados secundários foram analisados, tais como: indicadores de avaliação da Programação da Atenção da Vigilância em Saúde (PAVS); resolução e portarias; o Diário Oficial do estado da Bahia no período de 1999 a 2001; e, a ata de reunião da Comissão Intergestora Bipartite para a análise do contexto. Nos estudos de caso foram analisados o Plano Municipal de Saúde (PMS), o Relatório de Gestão Municipal e os dados do Sistema de Informação de Agravos Notificáveis.

O plano de indicadores contemplou as três dimensões da gestão contendo os indicadores, os parâmetros, as fontes e os meios de verificação e as premissas. Para a análise dos indicadores quantitativos foi construído o índice global padronizado, que resultou da combinação (média aritmética) dos escores padronizados do índice 0 (obtido através de dados secundários) e do índice 1 (resultante dos indicadores definidos para cada dimensão da capacidade de gestão da VE).

O índice 1 foi gerado mediante a proporção das respostas afirmativas dadas pelos gestores a cada uma das dimensões. Posteriormente, os indicadores foram padronizados em $\mathrm{z}$ escore, para ficar na mesma escala do índice 0 , que foi gerado pela combinação de alguns indicadores de saúde dos municípios. Desta forma, para cada município foi atribuído um escore médio padronizado para a dimensão organizacional, operacional e da sustentabilidade e para os indicadores de saúde. Portanto, o índice 1 padronizado corresponde à média aritmética dos três indicadores e do índice 0 .

O índice global foi categorizado através dos quartis, resultando em quatro categorias definidas como muito ruim, ruim, regular e boa. De posse desta classificação foram selecionados os dois municípios-caso. Esta mesma categorização foi também aplicada ao índice 0 , a fim de agrupar os municípios nas categorias definidas.

As perdas em relação à amostra dos municípios $(n=108)$ foram assumidas como aleatórias, após a avaliação de que não ocorreram diferenças estatisticamente significantes entre o grupo dos municípios que responderam ao questionário, denominados municípios amostrados $\left(\mathrm{n}_{1}=38\right)$, e o grupo de municípios que não responderam $\left(\mathrm{n}_{2}=70\right)$, denominados municípios não amostrados, quando se comparou o índice global padronizado, definido com base nos dados secundários bem como em cada um dos seus componentes.

Para a análise do contexto buscou-se a produção dos sentidos a partir das práticas discursivas dos entrevistados. Adotando-se as ideias de Spink e Lima ${ }^{7}$, foram construídas linhas narrativas consideradas apropriadas para esquematizar conteúdos históricos.

Para a análise dos casos, utilizou-se o mapa de associação de ideias, que se inicia pela definição das categorias gerais de natureza temática. Após esta definição, organizam-se os conteúdos procurando preservar as sequências das falas e manter intacto o diálogo ${ }^{7}$.

\section{Reconstituindo a história da descentralização da vigilância epidemiológica no estado da Bahia}

A descentralização da gestão da VE para os municípios no estado da Bahia apresenta algumas peculiaridades, identificadas nas entrevistas e nos documentos analisados.

Para o Gestor 1 (GE1) a descentralização das ações de imunização foi o marco para o início da descentralização da vigilância epidemiológica no estado, com a organização das salas de vacina e seu gerenciamento pelos municípios, com trabalhadores da Sesab colocados à disposição das secretarias municipais. Para este gestor a descentralização da VE antecede a Portaria no 1.339 do Ministério da Saúde, publicada no ano de 1999, a qual define competências das esferas de governo na área de vigilância e controle das doenças. Esta mesma Portaria se constituiu, segundo a maioria dos entrevistados, no principal mecanismo de indução das ações de vigilância epidemiológica pelos municípios, uma vez que regulamentou a NOB 01/96 quanto às competências de cada esfera de governo e definiu a sistemática de financiamento.

A mesma Portaria induziu a adesão dos gestores municipais aos termos de compromisso, na medida em que receberiam um aporte adicional de recursos financeiros. Esta adesão significava que o município assumia dispor da estrutura preconizada nas Resoluções CIB-BA de 2000 e 2001, quanto à equipe, à estrutura e ao elenco mínimo de ações, quase sempre inexistente, como revela também esta avaliação. 
As entrevistas e a análise dos documentos indicam que a CIB/BA, como afirma Guimarães ${ }^{8}$, funciona como espaço para implementação do processo de certificação, através da conferência dos requisitos apresentados pelos municípios para habilitar-se a gestores da VE em seus territórios. O Gestor 3 afirma que foi difícil o processo de implantação da descentralização da VE para os municípios, na medida em que estes assinavam os termos de compromissos informando possuir toda a estrutura definida pela Resolução $n^{\circ}$ 27/2001, quando a realidade era outra, posto que faltavam veículos, estrutura física adequada e profissionais, particularmente de nível superior.

Apesar do discurso do Gestor 3 apontar para um acordo com os municípios na implantação do processo de descentralização da VE, identifica-se o modo centralizado desse processo, na medida em que foi direcionado através de portarias e resoluções, sem observar as especificidades locais.

Por fim, o Gestor 3 identifica que as dificuldades encontradas podem estar relacionadas ao compromisso e ao entendimento dos gestores quanto à importância da VE. Essa narrativa apóia os resultados do estudo de Santos ${ }^{9}$, Santos e Melo ${ }^{10}$ e Santos et al. ${ }^{11}$ quando os atores desta pesquisa concebem a VE como ação focada no controle das doenças transmissíveis e como poder de policia médica e, episodicamente, como monitoramento dos risco e danos da saúde da população.

Identifica-se também que a descentralização da VE na Bahia somente toma impulso a partir do ano 2000, destacando-se como fato marcante para o GE 1 o processo de negociação entre a Sesab e a Fundação Nacional de Saúde quanto à alocação dos trabalhadores e dos equipamentos. Com esse processo de negociação, que durou aproximadamente seis meses, a descentralização da gestão da VE, mediante certificação dos municípios, aconteceu entre 2001 e 2002.

Já o Gestor 2 afirma que o pouco tempo em que trabalha na Sesab não lhe permite situar historicamente a descentralização. Isso pode sinalizar que as pessoas que ocupam funções gestoras na VE não conhecem o contexto da sua área de atuação, ora porque não existem medidas institucionais para situá-los, ora porque, para aquele ator, conhecer como se processou a descentralização da VE não é significativo para o seu trabalho. Para este gestor, a descentralização da VE é concebida como desconcentração de atividades, com as diretorias regionais de saúde assumindo a supervisão das ações desenvolvidas pelos municípios, com vistas ao cumprimento de metas.
O Gestor 4 situa a descentralização da VE na Bahia como a mais atrasada do País, apesar de utilizar uma linguagem verbal não explícita. Essa narrativa guarda coerência com achados do estudo de Guimarães ${ }^{8}$, atribuindo este atraso em decorrência da hegemonia política do grupo liderado por Antonio Carlos Magalhães.

O Gestor 5 destaca que a descentralização incipiente da VE também se justifica pelo despreparo da equipe e porque alguns municípios não aderiram a esse processo. Diante dessa afirmação surge uma indagação: como poderiam os municípios ser inicialmente competentes para desenvolver as ações de Vigilância Epidemiológica, quando historicamente esta era uma competência do estado?

Para o Gestor 5, apesar da existência de arcabouço legal, era necessário um investimento em capacitações para que a equipe local realizasse as ações. Mesmo se mostrando favorável à descentralização, o discurso apresenta certo paradoxo, pois de um lado indica apoio ao processo de descentralização, ao mesmo tempo em que sempre reforça o acompanhamento e o gerenciamento das ações pelo estado, principalmente nas ações de imunização.

Por fim, o Gestor 6 explicita o caráter de serviço de inteligência da VE, quando identifica mudanças nos fatores determinantes e condicionantes da saúde individual e coletiva. No entanto, o desenvolvimento das ações de VE nos municípios é atribuído ao compromisso individual do técnico responsável pelas ações.

Nota-se que a normativa autonomia gerencial e financeira que os municípios adquiriram para desenvolver as ações de VE não elimina a deficiência de recursos, não somente de materiais, de estrutura física ou de equipamentos. A insuficiente qualificação dos trabalhadores para atuar no serviço da VE também constrange essa autonomia. Portanto, mesmo diante do arcabouço legal da descentralização da gestão da VE, verifica-se que não existe compartilhamento no processo decisório.

A convergência dos discursos dos gestores indica a existência de um processo vertical na descentralização da VE no estado da Bahia, característica predominante nas políticas públicas no Brasil.

\section{Resultados da linha de base avaliativa}

A linha de base avaliativa expressa em que medida um programa ou uma intervenção, dentro de um determinado contexto, atingiu os objetivos propostos. 
Observa-se que 50\% dos municípios apresentam uma capacidade de gestão da VE entre muito ruim e ruim, contra $26,3 \%$ dos municípios que apresentam uma capacidade de gestão da VE regular e 23,7\% dos municípios que configuram uma boa capacidade de gestão de VE.

Quando se avalia as macrorregiões separadamente, e tomando como referência o índice geral (combinação do índice 0 com os indicadores da dimensão organizacional, operacional e de sustentabilidade da gestão), verifica-se que a macrorregião Leste encontra-se em melhor situação, tendo em vista que nenhum município se inseriu no quartil entre muito ruim e ruim. Nessa macrorregião 33,3\% dos municípios tem a capacidade de gestão avaliada como regular e $66,7 \%$ como boa. Em situação inversa encontrase a macrorregião Nordeste, pois a maioria dos municípios obteve avaliação entre muito ruim $(33,3 \%)$ e ruim $(50 \%)$.

Depreende-se que o grau diferenciado de recursos, e consequentemente de poder, configura-se como elementos que podem dificultar a gestão da VE nos municípios. O modelo de organização dos serviços de saúde, incluídos os serviços de VE, reproduz as desigualdades entre as macrorregiões e os municípios, determinadas em função do seu grau de desenvolvimento social e econômico.

Numa avaliação geral da dimensão operacional, observa-se que os municípios possuem uma capacidade regular (71\%), e uma capacidade muito ruim $(26,3 \%)$ de manter e mobilizar recursos que garantam condições desejáveis de gestão, de modo a executar as ações de VE que se traduzam em melhoria dos indicadores de saúde da população.

Quanto à dimensão organizacional, 39,5\% dos municípios apresentam uma avaliação muito ruim, contra $26,3 \%$ com boa avaliação, evidenciando uma relação ainda frágil da autonomia decisória das instâncias locais, reforçando a perspectiva de que a descentralização da gestão da VE assume um caráter mais normativo do que político, e é, portanto, uma desconcentração de ações e atividades.

No que se refere à dimensão da sustentabilidade, observa-se que 50\% dos municípios foram avaliados como ruim e muito ruim, e os outros $50 \%$ avaliados entre regular e bom, permitindo inferir que, assim como a dimensão organizacional, ainda são frágeis os mecanismos e estratégias de gestão que ampliem ou fortaleçam as parcerias e as alianças para a sustentabilidade das decisões e dos resultados nos municípios.
Os resultados coincidem com os estudos de Barreto e Guimarães ${ }^{4}$ e de Landim ${ }^{12}$ quanto à fragilidade da autonomia decisória e da capacidade para definir diretrizes políticas pelos municípios. Estes possuem uma autonomia técnica vulnerável quando se analisa a gestão de pessoas, devido à precarização das relações de trabalho e dos vínculos empregatícios, e também da fragilidade dos processos de educação permanente; dos processos de planejamento ainda pouco participativos; da inconsistência da avaliação das ações; da estrutura física e dos equipamentos deficientes e da escassez e rotatividade de pessoal, que dificultam a realização das ações; a gestão assume uma natureza mais administrativa que estratégica e política. No entanto, as alianças e as parcerias construídas, ainda que timidamente, podem indicar progresso nas práticas de gestão.

Diante do exposto, pode-se deduzir que as conclusões de Matus ${ }^{2}$ e Guimarães et al. ${ }^{3}$ fortalecem os resultados deste estudo, quando afirmam que a capacidade de governo (dimensão operacional) condiciona e é condicionada pelo projeto de governo (dimensão organizacional) e pela governabilidade (dimensão da sustentabilidade). Portanto, apropriando-se das palavras de Melo et al. $^{13}$, a capacidade de gestão da VE se revela frágil no estado, pois não apresenta o equilíbrio das três dimensões de gestão, dado que estas são interligadas e sua separação é apenas um recurso metodológico da avaliação.

\section{Resultados dos estudos de caso em dois municípios baianos}

Para responder por que os municípios baianos possuem graus diferenciados de capacidade de gestão da VE foram selecionados dois municípios casos, com as seguintes características:

Município 1: melhor capacidade de gestão da VE na linha de base avaliativa. Situa-se na Região Metropolitana de Salvador, inserido na macrorregião Leste do estado da Bahia. Tem uma população de 16.783 habitantes ${ }^{14}$, sua base econômica é a indústria e o PIB corresponde a R\$ 154 milhões.

Município 2: com frágil capacidade de gestão da VE na linha de base avaliativa. Situa-se no Centro Sul Baiano, macrorregião Sudoeste. Com uma população de 17.210 habitantes ${ }^{14}$, seu PIB é de 55 milhões e sua base econômica se fundamenta na pecuária e na agricultura.

$\mathrm{Na}$ análise dos achados dos dois casos estudados utilizou-se a técnica do mapa de associação de ideias, que sistematiza o processo de aná- 
lise das práticas discursivas a partir de categorias analíticas pré-definidas ${ }^{7}$ :

1. Dimensão organizacional: competências e habilidades do gestor; processo decisório; controle social; mecanismos formais de comunicação.

2. Dimensão operacional: recursos operacionais; gestão de pessoas; educação permanente; capacidade técnica; descentralização da VE para a equipe de saúde da família.

3. Dimensão da sustentabilidade: parcerias e alianças; acesso aos serviços de saúde; planos e projetos; recursos financeiros.

Vale destacar que na linha de base avaliativa o município com a melhor capacidade de gestão na VE obteve uma avaliação regular na dimensão operacional e da sustentabilidade e boa na dimensão organizacional. Já o município com a capacidade de gestão mais frágil tem uma avaliação ruim na dimensão organizacional, regular na dimensão operacional e muito ruim na dimensão da sustentabilidade. Os discursos dos sujeitos envolvidos no estudo ratificam em parte esta avaliação, através dos achados demonstrados nos mapas de associação de ideias e pela análise dos documentos.

A despeito das diferenças e semelhanças observadas, a avaliação da dimensão organizacional não atingiu um grau avançado em nenhum dos dois municípios analisados. O município 1 apresenta uma melhor situação (avaliação regular), ratificando em parte os resultados apresentados na linha de base avaliativa, e o município 2 um situação ruim, quanto à estrutura do processo decisório.

Analisando-se as falas dos entrevistados e os dados obtidos através do questionário on line, pode-se inferir que o município 1 encontra-se mais organizado do que o município 2 no que diz respeito aos mecanismos de mobilização social. O município 1 conta com um comitê de mobilização contra dengue, envolvendo vários segmentos da sociedade civil organizada, enquanto que no município 2 este mecanismo inexiste. Quanto ao CMS, os discursos dos entrevistados e os questionários confirmam as características insuficientes da capacidade de gestão da VE.

Percebe-se, nos dois municípios, que os gestores preocupam-se em desenvolver os programas instituídos pelo governo federal e estadual, ao invés de formular e operar políticas de saúde específicas.

Considerando que a dimensão organizacional da VE revela a capacidade de decidir de forma autônoma, participativa e transparente, os resultados encontrados apontam para situações de constrangimentos nos dois municípios, e mais acentuadamente no município 2 .

Analisando-se o número de unidades de saúde cadastradas e os profissionais existentes nos dois municípios, observa-se que é desigual a distribuição dos recursos: o município 1 conta com 22 unidades e 383 trabalhadores e o município 2 possui 19 unidades e 131 trabalhadores. Analisando-se os indicadores relativos ao PIB, IDH e IDI, nota-se que o município 1 apresenta uma condição econômica e social melhor do que o município 2, o que certamente contribui para a manutenção e a ampliação de recursos.

Em relação ao Piso Fixo de Vigilância e Promoção da Saúde (PFVPS) observa-se que os municípios da área metropolitana recebem valores superiores aos demais. Ou seja, a liberação de recursos federais para a capital e para os municípios que compõe sua região metropolitana é equivalente a, no mínimo, $80 \%$ do per capita do PFVPS; os demais municípios recebem valores equivalentes a, no mínimo, $60 \%$ do per capita do PFVPS atribuído ao estado correspondente.

O gestor da VE do município 1 atribui maior facilidade na gestão pelo tamanho pequeno deste e não pelo maior aporte financeiro recebido. No entanto, pode-se afirmar que existe uma correlação entre a capacidade de gestão e o porte econômico do município, o que por sua vez explica o melhor perfil do gestor no município 1 , dado que este pode manter profissionais com maior qualificação e em maior número.

Quando avaliada a capacidade técnica utilizando-se dos indicadores pactuados, observa-se um desempenho positivo no município 1 e regular no município 2 , sendo que em nenhum deles foi observado iniciativas na VE para além do que é determinado pelo Ministério da Saúde.

É na dimensão operacional que os municípios apresentam resultados semelhantes na linha de base avaliativa. Nos estudos de casos, no entanto, observa-se que a avaliação do município 1 expressa uma condição melhor do que o município 2 para executar as decisões. Este melhor desempenho na dimensão operacional do município 1 vincula-se ao perfil de alguns gestores intermediários (VE e atenção básica) e dos seus trabalhadores.

$\mathrm{Na}$ dimensão da sustentabilidade de gestão, conforme a avaliação na linha de base, a maior parte dos resultados é ruim, sendo que o município 1 obteve uma avaliação regular e o município 2 uma avaliação muito ruim. A análise dos discursos e dos documentos confirma este resultado, demonstrando mais uma vez que o municí- 
pio 1 encontra-se em melhor situação do que o município 2 no que se refere à capacidade de sustentar os resultados de gestão.

Observam-se algumas tentativas na construção de alianças e parcerias. No entanto, nos dois municípios é perceptível a reduzida capacidade de articulação intersetorial, em particular no município 2. Existe ainda um longo caminho a percorrer na busca de condições estruturais que viabilizem a intersetorialidade nas ações de saúde. As condições consideradas importantes para a construção de alianças estratégicas entre os diferentes setores governamentais e não governamentais não foram mencionadas nas entrevistas e nos documentos analisados.

A diferença na disponibilidade de recursos financeiros entre os municípios estudados é muito acentuada. O município 1 apresenta um percentual de receita própria destinado para a saúde de $22,3 \%$, e o município 2 um percentual de $23,2 \%$. Analisando-se esse dado isoladamente, pode-se afirmar que os municípios destinam um percentual de receita própria adequada aos requisitos da EC 29. Mas, quando se compara a receita do município 1 com a do município 2 , as diferenças emergem, pois o valor da receita do município 1 é sete vezes maior do que o do município 2 . O total de gasto por habitante no município 1 é cinco vezes maior do que o total gasto por habitante no município 2.

As evidências apontam que a sistemática de financiamento federal, apesar de igualar valores per capita, com distribuição de recursos através dos blocos de financiamento para a atenção básica; média e alta complexidade da assistência; vigilância em saúde; assistência farmacêutica e gestão, não leva em conta as desigualdades interregionais. Ainda que tenham porte populacional semelhante, o município 1 possui uma maior capacidade de gasto do que o município 2 , o que, somado a uma maior autonomia no processo decisório, lhe confere uma melhor capacidade de gestão da VE .

A análise dos dois casos revela fragilidade na capacidade de gestão da VE. O município 1 apresenta uma condição mais avançada do que o município 2 , mas não se observa equilíbrio entre as dimensões avaliadas. Houve convergência dos resultados quanto à pequena participação dos gestores da VE no processo decisório nos municípios, visto que estes se limitam a operacionalizar os programas instituídos pelas outras esferas de governo. Da mesma forma, o processo decisório no interior do SUS municipal não é horizontal, dado que a participação do CMS nas de- cisões relacionadas à gestão da VE não é efetiva, e os trabalhadores da saúde limitam-se a repassar as suas demandas para os níveis hierárquicos superiores. Não é institucionalizada a participação do CMS na elaboração de planos e projetos para a VE, inserindo o processo de descentralização numa dimensão mais técnica do que política, distanciando-se da concepção de descentralização assumida neste estudo.

Em relação ao planejamento, tanto no município 1 como no município 2, os PMS não foram destacados como instrumento de gestão, constituindo-se em instrumentos normativos para cumprimento de requisito estabelecido pelo $\mathrm{Mi}$ nistério da Saúde. Da mesma forma, o relatório de gestão e a programação operativa anual, também instrumentos implantados por iniciativa do Ministério da Saúde para subsidiar a avaliação das ações propostas no PMS, não são elaborados com esse fim, em particular no município 2, onde a avaliação dos indicadores de saúde e do financiamento foi elaborada com uma defasagem de dois anos. É evidente a reduzida capacidade dos municípios para avaliar os dados gerados nos sistemas de informações e produzir informes epidemiológicos e boletins sobre o estado de saúde da população.

As maiores diferenças na avaliação recaem no aporte de recursos operacionais, pois o município 1 tem trabalhadores da saúde em quantidade e qualidade, além de um aporte financeiro, superior ao município 2, o que pode contribuir para um melhor desempenho da gestão da VE.

A sustentabilidade da gestão no município 1 é conferida em parte pela autonomia da secretaria municipal da saúde em relação à utilização e à disponibilidade de recursos financeiros para desenvolver as ações da VE. Em contrapartida, no município 2 o secretário municipal da saúde não tem a mesma autonomia, visto que as deliberações quanto ao gasto com os recursos são controlados pelo prefeito, além de contar com uma escassez destes para desenvolver as ações sob sua responsabilidade.

Observou-se, assim como apontado por Melo et $\mathrm{al}^{13}$, que o grau diferenciado de poder e de recursos, a autonomia relativa dos municípios e a superposição de competências fazem com que a descentralização da VE não promova um processo de gestão pactuada e compartilhada. A dependência de recursos financeiros e a falta de autonomia dos municípios em relação à esfera estadual e federal, evidenciada neste estudo, não se limita a um problema de arranjo institucional ou de redefinição de competências, uma vez que 
existem outras razões que explicam a ausência de poder local.

Assim, os estudos de caso revelam fragilidade nas três dimensões da gestão avaliada. No entanto, a dimensão operacional, a qual obteve uma melhor avaliação, poderá ficar comprometida no longo prazo, pois se observa constrangimentos no processo decisório; limitações na formação de alianças e de parcerias e no estabelecimento de canais de ausculta que permitam a identificação das necessidades de saúde da população local.

Ao relacionar os achados da avaliação com o princípio da descentralização assumido nesta pesquisa constata-se que o que existe nos municípios estudados é uma desconcentração das atividades, visto que a responsabilidade pela execução das ações de VE é atribuída pelas outras esferas de governo sem o correspondente compartilhamento de poder decisório.

\section{Considerações finais}

Os resultados desta avaliação apontam para acentuada fragilidade na capacidade de gestão da VE nos municípios. Tanto na linha de base avaliativa como nos estudos de caso, os resultados obtidos em todas as dimensões, notadamente na organizacional e na da sustentabilidade, apontam para constrangimentos na capacidade de gestão.

A análise do contexto revela que os municípios possuem pouca autonomia no processo decisório face ao caráter vertical e normativo que assume a descentralização da gestão da VE. A capacidade de gestão da VE encontra-se diretamente relacionada ao contexto da implantação da sua descentralização, marcado pela regulação do Estado através de leis, portarias e resoluções definindo competências, estrutura e financiamento da VE municipal, sem considerar as especificidades locais. Portanto, há mais constrangimentos do que compartilhamento de decisões, seja em relação a outras esferas de governo, seja no interior do SUS municipal.

Destaca-se com melhor desempenho na avaliação a macrorregião Leste, área com melhor situação econômica e financeira, evidenciando que as desigualdades existentes entre as macrorregiões estão relacionadas ao seu grau de desenvolvimento econômico e social. O município desta macrorregião tem indicadores econômicos e sociais que o coloca em posição privilegiada no ranking nacional e estadual.

$\mathrm{Na}$ dimensão organizacional chama atenção a falta de autonomia no processo decisório, em particular a do município 2, em função da centralização das decisões na figura do seu gestor máximo, além da incipiente participação do CMS no planejamento e na avaliação das ações. Não se considera, na seleção dos gestores, a qualificação e a experiência para atuar tanto como secretário municipal de saúde quanto como gestor intermediário. Destes, $42 \%$ não possuem experiência prévia em gestão e 37\% dos gestores da VE não possuem especialização na área de saúde. Tais achados, articulados à inexistência de critérios técnicos-políticos explícitos na escolha dos gestores municipais, incluídos os da VE, limita a capacidade de gestão neste âmbito de prestação de serviços.

$\mathrm{Na}$ dimensão operacional as maiores fragilidades aparecem nos processos educativos, que geralmente não emergem a partir da identificação de necessidades locais; nas precárias relações de trabalho, com predomínio de contratos temporários, o que não assegura os direitos dos trabalhadores e impossibilita a qualificação de um quadro técnico competente e responsável para atuar na VE. Isto favorece a descontinuidade das ações; diminui a capacidade de execução das atividades de VE; não agrega valor para a capacidade de gestão e tampouco para a legitimidade do gestor.

Os dados revelam que o foco da gestão da VE é o controle das doenças transmissíveis, através de ações pontuais, que se sobrepõem às ações de promoção da saúde. Predomina uma racionalidade técnica que enfatiza a busca de resultados a partir de metas previamente definidas pela Sesab e pelo Ministério da Saúde.

$\mathrm{Na}$ dimensão operacional se encontram os melhores resultados na linha de base avaliativa, assim como nos estudos de caso (avaliação regular). Tal fato pode estar relacionado com a capacidade técnica e o compromisso dos gestores intermediários e dos trabalhadores que desenvolvem as ações de VE, e menos pela compreensão dos gestores máximos da saúde e dos prefeitos quanto à posição estratégica da VE para a mudança do modelo de atenção e para a consolidação do SUS.

$\mathrm{Na}$ dimensão da sustentabilidade da gestão são frágeis as iniciativas para estabelecer parcerias e alianças, especialmente quanto ao financiamento das ações que possam sustentar no médio e longo prazo os resultados obtidos na dimensão operacional. É evidente a incipiente capacidade de formação de parcerias e alianças, e estas, quando existentes, são feitas com instâncias institucionais já parceiras.

Deste modo, é necessário rever critérios de financiamento das ações da vigilância em saúde, 
dado que os resultados indicam que o modelo atual promove a iniquidade, pois não possibilita ao município com frágil sustentação econômica desenvolver as inúmeras ações de VE.

Destacam-se, contudo, avanços em alguns indicadores de saúde advindos da descentralização da gestão da VE, em particular aqueles relacionados à ampliação da cobertura vacinal e à redução das doenças imunopreveníveis.

Assim, pode-se afirmar que não se verifica, de fato, uma gestão descentralizada da VE nos municípios avaliados. Existe uma expressiva variação na capacidade de gestão das macrorregiões e dos municípios, evidenciada na construção da linha de base avaliativa e nos estudos de caso, que é determinada pelas desigualdades sociais, econômicas, políticas e pelos mecanismos de gestão adotados.

\section{Colaboradores}

SSBS Santos elaborou o artigo a partir da sua tese de doutorado; CMM Melo, orientadora da referida tese, contribuiu na concepção e foi responsável pela revisão critica final do artigo; HOG Costa revisou a versão final do artigo e participou da elaboração da metodologia da pesquisa; OY Tanaka participou da validação da metodologia e revisou o artigo; FM Ramos, MCC Santana e BG Trindade participaram da coleta de dados da tese e da discussão e da análise dos casos.

\section{Agradecimento}

À Capes pela bolsa de estudo e ao $\mathrm{CNPq}$ pelo financiamento da pesquisa e da tese de doutorado que dá origem ao artigo. 


\section{Referências}

1. Lemieux V. Décentralisation, politiques publiques et relations de pouvoir. Montreal: Les Presses de l'Université de Montreal; 2001.

2. Matus C. Política, planejamento e governo. Brasília: IPEA; 1993.

3. Guimarães MCL, Santos SMC dos, Melo C, Sanches Filho A. Avaliação da capacidade de gestão de organizações sociais: uma proposta metodológica em desenvolvimento. Cad Saude Publica 2004; 20(6):1642-1650.

4. Barreto JL, Guimarães MCL. Avaliação da gestão descentralizada da assistência farmacêutica básica em municípios baianos, Brasil. Cad Saude Publica 2010; 26(6):1207-1220.

5. Brasil. Ministério da Saúde (MS). Lei Orgânica da Saúde no ${ }^{\circ} 8080$ de 19 de setembro de 1990. Dispõe sobre as condições para promoção, proteção e recuperação da saúde, a organização e o funcionamento dos serviços correspondentes e dá outras providências. Diário Oficial da União 1990; 20 set.

6. Minayo MCS, Assis SG, Souza ER, organizadoras. Avaliação por triangulação de métodos: abordagens de programas sociais. Rio de Janeiro: Ed. Fiocruz; 2005.

7. Spink MJP, Lima H. Rigor e visibilidade: a explicitação dos passos da interpretação. In: Spink MJP, organizadora. Práticas discursivas e produção de sentidos no cotidiano: aproximações teóricas e metodológicas. São Paulo: Cortez; 1999. p. 93-122.

8. Guimarães MCL. Descentralização da saúde, interesses e conflitos decisórios: o processo de decisão nas instâncias colegiadas estaduais, Bahia, 1993-1998. [tese]. Salvador (BA): Universidade Federal da Bahia; 2000.
9. Santos SSBS. Avaliação da descentralização da vigilância epidemiológica para a equipe de saúde da família. [dissertação]. Salvador (BA): Universidade Federal da Bahia; 2006.

10. Santos SBSS, Melo CMM de. Avaliação da descentralização da vigilância epidemiológica para a Equipe de Saúde da Família. Cienc Saude Colet 2008; 13(6):1923-1932.

11. Santos SSBS, Melo CMM, Perrault M. Idéologies des presonnels de santé dans les conceptions de la surveillance épidémiologique. Revue Pratique et organization de soins 2010; 41(2):143-149.

12. Landim ELAS. Descentralização da gestão do Programa de DST/AIDS no Estado da Bahia: desafios para o município como território de práticas de saúde. [dissertação]. Salvador (BA): Universidade Federal da Bahia; 2008.

13. Melo CMM, Costa HOG, Santana G, Santos H. Avaliação da capacidade de gestão terceirizada de unidades de saúde do SUS municipal. Relatório técnico final. Salvador: Grupo de Pesquisa Gerir, Escola de Enfermagem da Universidade Federal da Bahia. Salvador, 2009.

14. Instituto Brasileiro de Geografia e Estatística (IBGE). Informações estatísticas 2007. [site da Internet]. [acessado 2010 ago 10]. Disponível em: http://www.ibge. gov.br/cidadesat/topwindow.htm

Artigo apresentado em 10/10/2011

Aprovado em 25/11/2011

Versão final apresentada em 08/12/2011 